\title{
Dynamics of Endomorphism of Finite Abelian Groups
}

\author{
Zhao Jinxing ${ }^{1,2}$ and Nan Jizhu ${ }^{1}$ \\ ${ }^{1}$ School of Mathematical Sciences, Dalian University of Technology, Dalian 116024, China \\ ${ }^{2}$ School of Mathematical Sciences, Inner Mongolia University, Hohhot 010021, China \\ Correspondence should be addressed to Zhao Jinxing; zhjxing@imu.edu.cn
}

Received 16 April 2017; Accepted 11 July 2017; Published 8 August 2017

Academic Editor: Allan C. Peterson

Copyright (c) 2017 Zhao Jinxing and Nan Jizhu. This is an open access article distributed under the Creative Commons Attribution License, which permits unrestricted use, distribution, and reproduction in any medium, provided the original work is properly cited.

We study the dynamics of endomorphisms on a finite abelian group. We obtain the automorphism group for these dynamical systems. We also give criteria and algorithms to determine whether it is a fixed point system.

\section{Introduction}

A discrete dynamical system is consisting of a pair $(X, f)$, where $X$ is a nonempty set and $f: X \rightarrow X$ is a function. The functional graph of $(X, f)$ is a directed graph with vertex set $X$ and an arrow from $x$ to $y$ if and only if $f(x)=y$. We denote the functional graph of $(X, f)$ by $G(X, f)$.

Finite discrete dynamical systems appeared in engineering, control systems computer science, and genetic networks; see $[1,2]$. One of the most important examples of discrete dynamical systems is given by $\left(\mathbb{F}_{q}^{n}, f\right)$, where $\mathbb{F}_{q}^{n}$ is the vector space over a finite field $\mathbb{F}_{q}$ and $f$ is a linear transformation. The structure these dynamical systems can be described by the elementary divisors of the corresponding matrix; see $[3,4]$. By using the same method, Brown and Vaughan [5] studied the cycle structure of linear dynamical system over $\mathbb{Z}_{n}$. Bach and Bridy [6] gave an upper and a lower bound for the distinct number of functional graph defined by affine mapping over finite dimensional linear space over finite fields. But the study of linear dynamical systems over a general finite commutative ring $R$ has difficulties since the factorization of polynomials in $R[x]$ is not unique. Brown and Vaughan [5] showed that $R^{n}$ can be decompose into $R^{n}=$ ker $f^{k} \oplus \operatorname{Im} f^{k}$ by Fitting's Lemma and $\left(R^{n}, f\right)$ is the product of the dynamical systems induced on $\operatorname{ker} f^{k}$ and $\operatorname{Im} f^{k}$. They also provided an algorithm to compute the minimal positive integer $k$ in the above decomposition, but the algorithm is not efficient. Xu and Zou [7] obtained an efficient algorithm to compute $k$ under a reasonable assumption on the size of the ring $R$. Deng [8] gave a criterion to determine whether a linear dynamical system is over the ring of integer modulo $m$.

Let $H$ be a finite abelian group written additively and $\operatorname{End}(H)$ be the endomorphism ring of $H$. In this paper we are interested in the dynamical systems $(H, f)$ defined by $f \in$ $\operatorname{End}(H)$. These dynamical systems have nice structure and were studied in many special cases; see [9]. In this paper we are interested in the case in which $X$ is a finite abelian group $H$ written additive, and $f: H \rightarrow H$ is an endomorphism of $H$.

The paper is organized as follows. In Section 2, we give some notations and basic results on the structure of functional graphs $G(H, f)$. In Section 3, we obtain necessary and sufficient conditions for $G\left(H_{1}, f_{1}\right)$ and $G\left(H_{2}, f_{2}\right)$. We also give an explicit form of the automorphism group of $G(H, f)$. In Section 4, we obtain a criterion to determine whether $G(H, f)$ is a fixed point system.

\section{Elementary Properties of $G(H, f)$}

A component of a digraph is a maximal connected subgraph of the associated nondirected graph. The indegree of a vertex $a$ of $G(X, f)$, denoted by indeg $f_{X}^{f}(a)$, is the number of directed edges coming into $a$. The outdegree $\operatorname{outdeg}_{X}^{f}(a)$ of $a$ is the number of directed edges leaving the vertex $a$. It is clear that the outdegree of any vertex in $G(X, f)$ is equal to 1 , and the indegree of a vertex $a$ is the number of vertices coming to $a$. A vertex $x$ in $G(X, f)$ is called a cycle vertex if 
$f^{k}(x)=x$ for some positive integers $k$. In particular, a cycle vertex $x$ is called a fixed point of $G(X, f)$ if $f(x)=x$. Let $A_{t}(X, f)$ denote the number of $t$-cycles contained in $G(X, f)$. Let $\mathscr{A}(X, f)$ denote the set of positive integers which is the length of the cycle which appeared in $G(X, f)$. We need the following results about $A_{t}(X, f)$ and $\mathscr{A}(X, f)$. In particular, let $d(X, f)=|\{x \in X: f(x)=x\}|$ be the number of fixed point in $(X, f)$.

Definition 1. We define a height function on the vertices and components of $G(X, f)$. Let $a$ be a vertex of $G(X, f)$. Then we define $h(a)$ to be the minimal nonnegative integer $i$ such that $f^{i}(a)$ is a cycle vertex in $G(X, f)$. We set $h(D)=\sup _{a \in D} h(a)$ if $D$ is a component of $G(X, f)$.

Definition 2. For any integer $i \geq 0$ and any vertex $a \in$ $G(X, f)$, define

$$
\begin{aligned}
& \mathscr{F}^{0}(a)=\{a\}, \\
& \mathscr{F}^{i}(a)=\left\{b \mid f^{i}(b)\right. \\
&\left.=a, f^{i-1}(b) \text { is not a cycle vertex if } i>0\right\} .
\end{aligned}
$$

Let $T(a)$ be the subdigraph of $G(X, f)$ with the vertices set $\bigcup_{i=0}^{\infty} \mathscr{F}^{i}(a)$, and there is a directed arrow from $x$ to $y$ in $T(a)$ if $f(x)=y$ and $h(x)=h(y)+1$. Hence, $T(a)$ is a directed tree with root $a$.

Let $H$ be a finite abelian group and $f \in \operatorname{End}(H)$. The basic properties of $G(H, f)$ in this section are proved in some special cases; see [10-14]. We give some basic properties of these digraphs. The proof is similar, but we still present it.

Lemma 3. Let $f \in \operatorname{End}(H)$. Then

(1) the indegree of any vertex in $G(H, f)$ is 0 or $\mid$ ker $f \mid$.

(2) the number of the vertices with indegree $|\operatorname{ker} f|$ is $|H| /|\operatorname{ker} f|$.

Proof. Let $x$ be a vertex with positive indegree. Then $\{y$ : $f(y)=x$ is a coset of ker $f$ and they have the same number of elements. The statement (2) follows immediately from (1).

We present two results on the tree structure attached to a vertex.

Lemma 4. Let $c$ be a cycle vertex of $G(H, f)$. Then $T(c) \simeq$ $T(0)$.

Proof. For any $h>0$ there exists an unique cycle vertex $c_{h}$ such that $f^{h}\left(c_{h}\right)=c$ since $c$ is a cycle vertex. Now if $x \in \mathscr{F}^{h}(0)$, we define a map $\varphi_{h}: \mathscr{F}^{h}(0) \rightarrow \mathscr{F}^{h}(c)$ as follows:

$$
\varphi_{h}(x)=x+c_{h} .
$$

Then $f^{h}\left(x+c_{h}\right)=f^{h}(x)+f^{h}\left(c_{h}\right)=0+c=c$. We observe that $f^{h-1}(x)$ is not a cycle vertex, but $f^{h-1}\left(c_{h}\right)$ is a cycle vertex; then $f^{h-1}\left(x+c_{h}\right)$ is not a cycle vertex since the set of all cycle vertices of $G(H, f)$ is a subgroup of $H$. The map $\varphi_{h}$ is welldefined and clearly injective. If $b \in \mathscr{F}^{h}(c)$, then $b-c_{h} \in \mathscr{F}^{h}(0)$ and $\varphi_{h}\left(b-c_{h}\right)=b . \varphi_{h}$ is also surjective.

Now if $a \in \mathscr{F}^{h}(0)$ and $b \in \mathscr{F}^{h-1}(0)$ with $f(a)=b$, then

$$
\begin{aligned}
f\left(\varphi_{h}(a)\right) & =f\left(a+c_{h}\right)=f(a)+f\left(c_{h}\right)=b+c_{h-1} \\
& =\varphi_{h-1}(b) .
\end{aligned}
$$

Finally we set $\varphi: T(0) \rightarrow T(c):$

$$
\varphi(x)=\varphi_{i}(x),
$$

if $x \in \mathscr{F}^{i}(0)$. Вy (3) $\varphi$ is an isomorphism.

Corollary 5. Let $D_{1}$ and $D_{2}$ be two components of $G(H, f)$. Then $D_{1} \simeq D_{2}$ if and only if they have the same cycle length.

Proof. If follows immediately from Lemma 4.

Lemma 6. Let $H_{1}, H_{2}$ be two finite abelian groups and $f_{i} \in$ $\operatorname{End}\left(H_{i}\right)$. Let $c_{i} \in G\left(H_{i}, f_{i}\right)$ with positive height. Then $T\left(c_{1}\right) \simeq$ $T\left(c_{2}\right)$ if and only if $\left|\mathscr{F}^{j}\left(c_{1}\right)\right|=\left|\mathscr{F}^{j}\left(c_{2}\right)\right|$ for any $j \geq 0$.

Proof. Let $h_{i}=h\left(T\left(c_{i}\right)\right)$. First we assume that $T\left(c_{1}\right) \simeq T\left(c_{2}\right)$ and let $\varphi$ be an isomorphism. Since the length of the longest directed path in $T\left(c_{i}\right)$ is $h_{i}$ and this path ends at $c_{i}$, it follows that $\varphi\left(c_{1}\right)=c_{2}$. Thus, $\varphi\left(\mathscr{F}^{j}\left(c_{1}\right)\right) \subseteq \mathscr{F}^{j}\left(c_{2}\right)$ and $\left|\mathscr{F}^{j}\left(c_{1}\right)\right|=$ $\left|\mathscr{F}^{j}\left(c_{2}\right)\right|$.

Conversely, suppose that $\left|\mathscr{F}^{j}\left(c_{1}\right)\right|=\left|\mathscr{F}^{j}\left(c_{2}\right)\right|=n_{j}$ for any $j \geq 0$. Then $h=h_{1}=h_{2}$. We induct on $h$. The case $h=0,1$ is trivial. Note that for any vertex $a \in G\left(H_{i}, f_{i}\right)$ with positive height, by Definition $2\left|\mathscr{F}^{k}(a)\right|=\mid\left\{x \in H_{i}: f_{i}^{k}(x)=\right.$ $a\} \mid=\operatorname{indeg}_{H_{i}}^{f_{i}^{k}}(a)$. Since $f_{i}^{k}$ is also a group homomorphism, by Lemma 3 we have $\left|\mathscr{F}^{k}(a)\right|=n_{k}$ or 0 for any $k=0,1, \ldots, h$. Let $\mathscr{F}^{1}\left(c_{1}\right)=\left\{a_{1}, \ldots, a_{n_{1}}\right\}$ and $\mathscr{F}^{1}\left(c_{2}\right)=\left\{b_{1}, \ldots, b_{n_{1}}\right\}$. Let $x_{i}=\left|\left\{a \in \mathscr{F}^{1}\left(c_{1}\right): h(T(a))=i\right\}\right|$ and $y_{i}=\mid\left\{b \in \mathscr{F}^{1}\left(c_{2}\right):\right.$ $h(T(b))=i\} \mid$. Since $\mathscr{F}^{k}\left(c_{1}\right)=\bigcup_{a \in \mathscr{F}^{1}\left(c_{1}\right)} \mathscr{F}^{k-1}(a)$ is a disjoint union for any $k>1$ and $\left|\mathscr{F}^{k}(a)\right|=\left|\mathscr{F}^{k}\left(c_{1}\right)\right|=n_{k}$ if and only if $h(T(a)) \geq k$, one has $\left|\mathscr{F}^{k}\left(c_{1}\right)\right|=n_{k}=\sum_{a \in \mathscr{F}^{k-1}\left(c_{1}\right)}\left|\mathscr{F}^{k-1}(a)\right|=$ $n_{k-1}\left(x_{1}+\cdots+x_{k-1}\right)$. It follows that

$$
\begin{aligned}
& x_{0}+x_{1}+\cdots+x_{h-1}=n_{1} \\
& n_{1}\left(x_{1}+\cdots+x_{h-1}\right)=n_{2}
\end{aligned}
$$

$$
n_{h-1} x_{h-1}=n_{h}
$$

We derive that $x_{i}=n_{i+1} / n_{i}-n_{i+2} / n_{i+1}$ for $i \in\{0,1, \ldots, h-2\}$ and $x_{h-2}=n_{h} / n_{h-1}$. We obtain $y_{i}=x_{i}$ by replacing $c_{1}$ by $c_{2}$ in the above arguments. After reordering we may assume that $h\left(T\left(a_{i}\right)\right)=h\left(T\left(b_{i}\right)\right)$ for any $i$. Then $\left|\mathscr{F}^{j}\left(a_{i}\right)\right|=\left|\mathscr{F}^{j}\left(b_{i}\right)\right|$ for any fixed $i$ and $j$. By induction, there exists digraph isomorphism $\varphi_{i}: T\left(a_{i}\right) \rightarrow T\left(b_{i}\right)$. We set $\varphi: T\left(c_{1}\right) \rightarrow T\left(c_{2}\right)$, where $\varphi\left(c_{1}\right)=$ $c_{2}$ and $\varphi(z)=\varphi_{i}(z)$ if $z \in T\left(a_{i}\right)$. Then $\varphi$ is also a digraph isomorphism. This finishes the proof. 


\section{Automorphism Group of $G(H, f)$}

We first show when $G\left(H, f_{1}\right)$ is isomorphic to $G\left(H, f_{2}\right)$ for $f_{1}, f_{2} \in \operatorname{End}(H)$.

Lemma 7. Let $H_{1}, H_{2}$ be two finite abelian groups and $f_{i} \in$ End $\left(H_{i}\right)$. Let $D_{i}$ be the component of $G\left(H_{i}, f_{i}\right)$ containing the vertex 0 . Then $G\left(H_{1}, f_{1}\right) \simeq G\left(H_{2}, f_{2}\right)$ if and only if

(1) $D_{1} \simeq D_{2}$;

(2) $A_{t}\left(H_{1}, f_{1}\right)=A_{t}\left(H_{2}, f_{2}\right)$ for any $t$.

Proof. It follows immediately from Lemma 4 and Corollary 5.

Theorem 8. Let $H_{1}, H_{2}$ be two finite abelian groups and $f_{i} \in$ $\operatorname{End}\left(H_{i}\right)$. Then $G\left(H_{1}, f_{1}\right) \simeq G\left(H_{2}, f_{2}\right)$ if and only if for any positive integer $k$

(1) $\left|\operatorname{ker} f_{1}^{k}\right|=\left|\operatorname{ker} f_{2}^{k}\right|$;

(2) $\left|\operatorname{ker}\left(i d-f_{1}^{k}\right)\right|=\left|\operatorname{ker}\left(i d-f_{2}^{k}\right)\right|$.

Proof. Let $D_{i}$ be the component of $G\left(H_{i}, f_{i}\right)$ containing the vertex 0 . Since $x \in \operatorname{ker} f_{i}^{k}$ if and only if $f_{i}^{j}(x)=0$ for some $1 \leq$ $j \leq k$, so $\operatorname{ker} f_{i}^{k}=\bigcup_{j=0}^{k} \mathscr{F}^{j}\left(D_{i}\right)$ is a disjoint union. It follows that $\left|\operatorname{ker} f_{i}^{k}\right|=\sum_{j=0}^{k}\left|\mathscr{F}^{j}\left(D_{i}\right)\right|$ for any $k \geq 1$. Thus, $\left|\operatorname{ker} f_{1}^{k}\right|=$ $\left|\operatorname{ker} f_{2}^{k}\right|$ for any $k \geq 1$ if and only if $\left|\mathscr{F}^{k}\left(D_{1}\right)\right|=\left|\mathscr{F}^{k}\left(D_{2}\right)\right|$ for any $k \geq 1$. By Lemma 6 , condition (1) is equivalent to (1) of Lemma 7. Note that $\left|\operatorname{ker}\left(i d-f_{i}^{k}\right)\right|=\mid\left\{x \in H_{i}: f_{i}^{k}(x)=\right.$ $x\} \mid=\sum_{t \mid k} t A_{t}\left(H_{i}, f_{i}\right)$. Thus, condition (2) is equivalent to (2) of Lemma 7. The proof is finished by Lemma 7.

Let $H$ be a finite abelian group. By the structure of finite abelian group, we have

$$
H \simeq \bigoplus_{p} H^{p}
$$

where $p$ runs over the set of prime divisor of $|H|$ and $H^{p}$ is the unique Sylow $p$-subgroup of $H$. Moreover, if there is another factorization of $H \simeq \bigoplus F^{p}$ in this way, it must have $H^{p} \simeq F^{p}$ for each $p$.

We also recall the product of graphs. Let $G_{1}$ and $G_{2}$ be two digraphs. The digraph product $G_{1} \times G_{2}$ is the digraph whose vertices are the set of order pairs $\left(a_{1}, a_{2}\right)$, where $a_{i}$ is a vertex of $G_{i}$. There is a directed edge from $\left(a_{1}, a_{2}\right)$ to $\left(b_{1}, b_{2}\right)$ in $G_{1} \times G_{2}$ if and only if there is a directed edge from $a_{1}$ to $b_{1}$ in $G_{1}$ and there is a directed edge from $a_{2}$ to $b_{2}$ in $G_{2}$.

Lemma 9. Let $H$ be a finite abelian group and $f \in \operatorname{End}(H)$. Suppose that $|H|=n=\prod_{i=1}^{r} p_{i}^{e_{i}}$ is prime factorization. Then

$$
G(H, f) \simeq G\left(H^{p_{1}}, f\right) \times \cdots \times G\left(H^{p_{r}}, f\right) .
$$

Proof. It follows immediately from the fact that $H=H^{p_{1}} \oplus$ $H^{p_{2}} \oplus \cdots \oplus H^{p_{r}}$ and $f\left(H^{p}\right) \subseteq H^{p}$.

Theorem 10. Let $H_{1}, H_{2}$ be two abelian groups and $f_{i} \in$ $\operatorname{End}\left(H_{i}\right)$. Then $G\left(H_{1}, f_{1}\right) \simeq G\left(H_{2}, f_{2}\right)$ if and only if $G\left(H_{1}^{p}, f_{1}\right) \simeq G\left(H_{2}^{p}, f_{2}\right)$ for any prime $p$.
Proof. First we assume that $G\left(H_{1}^{p}, f_{1}\right) \simeq G\left(H_{2}^{p}, f_{2}\right)$. By Lemma 9, we have

$$
\begin{aligned}
G\left(H_{i}, f_{i}\right) \simeq & G\left(H_{i}^{p_{1}}, f_{i}\right) \times G\left(H_{i}^{p_{2}}, f_{i}\right) \times \cdots \\
& \times G\left(H_{i}^{p_{r}}, f_{i}\right) .
\end{aligned}
$$

So we obtain $G\left(H_{1}, f_{1}\right) \simeq G\left(H_{2}, f_{2}\right)$.

Conversely, suppose that $G\left(H_{1}, f_{1}\right) \simeq G\left(H_{2}, f_{2}\right)$. Let $g_{i, k}=i d-f_{i}^{k}$. By Theorem 8, $\left|\operatorname{ker} f_{1}^{k}\right|=\left|\operatorname{ker} f_{2}^{k}\right|$ and $\left|\operatorname{ker} g_{1, k}\right|=\left|\operatorname{ker} g_{2, k}\right|$ for any $k>0$. It follows that $\left|\operatorname{ker}\left(\left.f_{1}^{k}\right|_{H_{1}^{p}}\right)\right|=\left|\operatorname{ker}\left(\left.f_{2}^{k}\right|_{H_{2}^{p}}\right)\right|$ and $\left|\operatorname{ker}\left(\left.g_{1, k}\right|_{H_{1}^{p}}\right)\right|=$ $\left|\operatorname{ker}\left(\left.g_{2, k}\right|_{H_{2}^{p}}\right)\right|$. By Theorem 8 again, $G\left(H_{1}^{p}, f_{1}\right) \simeq G\left(H_{2}^{p}, f_{2}\right)$. The proof is finished.

Next we would determine the automorphism group of the digraph $G(H, f)$. We need the wreath product of groups; see $[4,15]$. We rewrite in a simple way. Let $G$ be a group and $K$ be a permutation group on $\{1, \ldots, n\}$; then the wreath product $G$ < $K$ is generated by the direct product of $n$ copies of $G$, together with the elements of $K$ action on these $n$ copies of $G$. The wreath product is associative, so we write $G_{1}<G_{2}<\cdots<G_{k}$ for $\left(\left(\left(G_{1}<G_{2}\right)>G_{3}\right) \prec \cdots>G_{k-1}\right)>G_{k}$.

Theorem 11 (see [16]). Let $G$ be a graph. Suppose that the connected components of $G$ consist of $n_{1}$ copies and $G_{1}, \ldots, n_{r}$ copies of $G_{r}$, where $G_{1}, \ldots, G_{r}$ are pairwise nonisomorphic. Then

$$
\operatorname{Aut}(G) \simeq\left(\operatorname{Aut}\left(G_{1}\right)<S_{n_{1}}\right) \oplus \cdots \oplus\left(\operatorname{Aut}\left(G_{r}\right)<S_{n_{r}}\right),
$$

where $S_{n}$ is the symmetric group on a finite set of order $n$.

By the above Theorem and Corollary 5 , we have

$$
\operatorname{Aut}(G(H, f)) \simeq \bigoplus_{i \in \mathscr{A}(H, f)} \operatorname{Aut}\left(X_{i}\right)>S_{A_{i}(H, f)},
$$

where $X_{i}$ is a component of $G(H, f)$ with cycle length $i$. Let $T$ be the component containing 0 in $G(H, f)$. Sha [9] showed that $\operatorname{Aut}\left(X_{i}\right) \simeq \operatorname{Aut}(T)<C_{i}$, where $C_{i}$ is the cyclic group of order $i$. Thus,

$$
\operatorname{Aut}(G(H, f)) \simeq \bigoplus_{i \in \mathscr{A}(H, f)} \operatorname{Aut}(T) \imath C_{i} \prec S_{A_{i}(H, f)} .
$$

But he did not obtain the structure of $\operatorname{Aut}(T)$ in general. We would determine the automorphism group of $T$.

Lemma 12. Letc $\in G(H, f)$ be a vertex with $h(c)>0$. Suppose that $h(T(c))=r$ and $\left|\operatorname{ker} f^{i}\right|=n_{i}$ for $i=1,2, \ldots, r$. Then

$$
\begin{aligned}
& \operatorname{Aut}(T(c)) \simeq S_{n_{1}-n_{2} / n_{1}+1} \\
& \oplus\left(\bigoplus_{1<i_{1}<i_{2}<\cdots<i_{t} \leq r-1} S_{n_{1}-n_{2} / n_{1}+1} \geq S_{x_{i_{1}}} \geq S_{x_{i_{2}}} \geq \cdots, S_{x_{i_{t}}}\right) \\
& \oplus\left(\bigoplus_{1=i_{1}<i_{2}<\cdots<i_{t} \leq r-1} S_{n_{1}} 2 S_{x_{i_{1}}} \geq S_{x_{i_{2}}} 2 \cdots<S_{x_{i_{t}}}\right),
\end{aligned}
$$

where $x_{i}=n_{i+1} / n_{i}-n_{i+2} / n_{i+1}$. 
Proof. By induction on $r$, let $y_{i}=\mid\left\{a \in \mathscr{F}^{1}(c): h(T(a))=\right.$ $i\} \mid$ for $i=0,1, \ldots, r-1$. Then $n_{j}-n_{j-1}=\left|\mathscr{F}^{j}(c)\right|=$ $\sum_{a \in \mathscr{F}^{1}(c), h(T(a)) \geq j-1}\left|\mathscr{F}^{j-1}(a)\right|=n_{j-1} \sum_{i=j-1}^{r-1} y_{i}$. Thus,

$$
\begin{gathered}
y_{0}+y_{1}+\cdots+y_{r-1}=n_{1} \\
n_{1}\left(y_{1}+\cdots+y_{r-1}\right)=n_{2}-n_{1} \\
\vdots \\
n_{r-1} y_{r-1}=n_{r}-n_{r-1} .
\end{gathered}
$$

We derive that $y_{0}=n_{1}-n_{2} / n_{1}+1$ and $y_{i}=x_{i}$ for $i \geq 1$. Let $T_{i}=$ $T\left(a_{i}\right)$ for $a_{i} \in \mathscr{F}^{1}(c)$ and $h\left(T\left(a_{i}\right)\right)=i$. By Lemma 6, we have $T(u) \simeq T\left(a_{i}\right)$ if $u \in \mathscr{F}^{1}(c)$ and $h(T(u))=i$. By Theorem 11 and by induction we have

$$
\begin{aligned}
& \operatorname{Aut}(T(c)) \simeq S_{y_{0}} \oplus\left(\bigoplus_{j=1}^{r-1} \operatorname{Aut}\left(T_{j}\right) \geq S_{x_{j}}\right) \\
& \simeq S_{y_{0}} \bigoplus_{j=1}^{r-1}\left(\bigoplus_{1<i_{1}<i_{2}<\cdots<i_{t} \leq j-1} S_{y_{0}}<S_{x_{i_{1}}} \geq S_{x_{i_{2}}}, \cdots<S_{x_{i_{t}}}\right) \\
& \text { ? } S_{x_{j}} \\
& \oplus\left(\bigoplus_{1=i_{1}<i_{2}<\cdots<i_{t} \leq j-1} S_{n_{1}}, S_{x_{i_{1}}}, S_{x_{i_{2}}}, \cdots, S_{x_{i_{t}}}\right) \\
& \text { ? } S_{x_{j}} \\
& \simeq S_{y_{0}} \oplus\left(\bigoplus_{1<i_{1}<i_{2}<\cdots<i_{t} \leq r-1} S_{y_{0}}>S_{x_{i_{1}}}>S_{x_{i_{2}}}, \cdots<S_{x_{i_{t}}}\right) \\
& \oplus\left(\bigoplus_{1=i_{1}<i_{2}<\cdots<i_{t} \leq r-1} S_{n_{1}}, S_{x_{i_{1}}}, S_{x_{i_{2}}}, \cdots, S_{x_{i_{t}}}\right) \text {. }
\end{aligned}
$$

Theorem 13. Suppose that $\left|\operatorname{ker} f^{i}\right|=n_{i}$ and $x_{i}=n_{i+1} / n_{i}-$ $n_{i+2} / n_{i+1}$. Then

$$
\begin{aligned}
\operatorname{Aut} & (T(0)) \simeq S_{n_{1}-n_{2} / n_{1}} \\
& \oplus\left(\bigoplus_{1<i_{1}<i_{2}<\cdots<i_{t} \leq r-1} S_{n_{1}-n_{2} / n_{1}+1} \geq S_{x_{i_{1}}}, S_{x_{i_{2}}}, \cdots<S_{x_{i_{t}}}\right) \\
& \oplus\left(\bigoplus_{1=i_{1}<i_{2}<\cdots<i_{t} \leq r-1} S_{n_{1}}, S_{x_{i_{1}}}, S_{x_{i_{2}}}, \cdots<S_{x_{i_{t}}}\right) .
\end{aligned}
$$

Proof. The proof is similar to Lemma 12. We induct on $r=$ $h(T(0))$. Let $z_{i}=\left|\left\{a \in \mathscr{F}^{1}(c): h(T(a))=i\right\}\right|$ for $i=$
$0,1, \ldots, r-1$. Note that $z_{0}+z_{1}+\cdots+z_{r-1}=n_{1}-n_{0}=n_{1}-1$ since 0 is a fixed point. Thus,

$$
\begin{gathered}
z_{0}+z_{1}+\cdots+z_{r-1}=n_{1}-1 \\
n_{1}\left(z_{1}+\cdots+z_{r-1}\right)=n_{2}-n_{1} \\
\vdots \\
n_{r-1} z_{r-1}=n_{r}-n_{r-1} .
\end{gathered}
$$

It follows that $z_{0}=n_{1}-n_{2} / n_{1}$ and $z_{i}=x_{i}$ for $i \geq 1$. Let $T_{i}=T\left(a_{i}\right)$ for $a_{i} \in \mathscr{F}^{1}(0)$ and $h\left(T\left(a_{i}\right)\right)=i$. By induction,

$$
\begin{aligned}
& \operatorname{Aut}(T(c)) \simeq S_{z_{0}} \oplus\left(\bigoplus_{j=1}^{r-1} \operatorname{Aut}\left(T_{j}\right) \prec S_{x_{j}}\right) \simeq S_{z_{0}} \\
& \cdot \bigoplus_{j=1}^{r-1}\left(\bigoplus_{1<i_{1}<i_{2}<\cdots<i_{t} \leq j-1} S_{y_{0}}, S_{x_{i_{1}}}, S_{x_{i_{2}}}, \cdots, S_{x_{i_{t}}}\right) \\
& 2 S_{x_{j}} \oplus\left(\bigoplus_{1=i_{1}<i_{2}<\cdots<i_{t} \leq j-1} S_{n_{1}}, S_{x_{i_{1}}}, S_{x_{i_{2}}}, \cdots<S_{x_{i_{t}}}\right) \\
& \imath S_{x_{j}} \simeq S_{n_{1}-n_{2} / n_{1}} \\
& \oplus\left(\bigoplus_{1<i_{1}<i_{2}<\cdots<i_{t} \leq r-1} S_{n_{1}-n_{2} / n_{1}+1}<S_{x_{i_{1}}}<S_{x_{i_{2}}} \geq \cdots<S_{x_{i_{t}}}\right) \\
& \oplus\left(\bigoplus_{1=i_{1}<i_{2}<\cdots<i_{t} \leq r-1} S_{n_{1}}, S_{x_{i_{1}}}, S_{x_{i_{2}}}, \cdots<S_{x_{i_{t}}}\right) .
\end{aligned}
$$

\section{Fixed Point Systems}

A dynamical system is called a fixed point system if its cycle vertices are all fixed points. In this section we would describe when $G(H, f)$ is a fixed point system.

Definition 14. Let $\left(X_{1}, f_{1}\right)$ and $\left(X_{2}, f_{2}\right)$ be two dynamical systems. A morphism $\varphi:\left(X_{1}, f_{1}\right) \rightarrow\left(X_{2}, f_{2}\right)$ is a map $\varphi: X_{1} \rightarrow X_{2}$ such that $f_{2} \varphi=\varphi f_{1}$.

Lemma 15. Let $\varphi:\left(X_{1}, f_{1}\right) \rightarrow\left(X_{2}, f_{2}\right)$ be a morphism of dynamical systems. Suppose that $\left(X_{1}, f_{1}\right)$ is a fixed point system and $\varphi$ is surjective. Then $\left(X_{2}, f_{2}\right)$ is also a fixed point system.

Proof. It follows immediately from Lemma 3.1 in [8].

Lemma 16. Let $H$ be a finite abelian group and $f \in \operatorname{End}(H)$. Let $f_{w}: H \rightarrow H, f_{w}(x)=f(x)+w$ be the affine mapping for any $w \in H$. Then

(1) $(H, f) \simeq\left(H, f_{w}\right)$ if and only if $\left(H, f_{w}\right)$ has a fixed point;

(2) $\left(H, f_{w}\right)$ is a fixed point system if and only if it has a fixed point and $(H, f)$ is a fixed point system. 
Proof. It follows immediately from Lemma 3.3 in [8].

The following lemma shows why we need to consider the affine system.

Lemma 17. Let $H_{1}, H_{2}$ be two finite abelian groups and $f_{i} \in \operatorname{End}\left(H_{i}\right)$. Suppose that $\varphi: H_{1} \rightarrow H_{2}$ is a group homomorphism and is also a surjective morphism of dynamical systems. Let $y_{0}$ be a fixed point in $\left(H_{2}, f_{2}\right)$ and $K=\varphi^{-1}\left(\left\{y_{0}\right\}\right)$. Then $\left(K,\left.f_{1}\right|_{K}\right) \simeq(\operatorname{ker} \varphi, g)$, where $g(x)=f_{1}(x)+f_{1}\left(x_{0}\right)-x_{0}$ for some $x_{0} \in K$.

Proof. It is clear that $K=x_{0}+\operatorname{ker} \varphi$ is a coset of $\operatorname{ker} \varphi$. We define $\psi: \operatorname{ker} \varphi \rightarrow K, \psi(a)=a+x_{0}$. Then $\varphi$ is bijective and $f_{1} \psi(a)=f_{1}\left(a+x_{0}\right)=f_{1}(a)+f_{1}\left(x_{0}\right)=\psi g(a)$. Thus, $\psi$ is an isomorphism of dynamical systems. $(X, f)$.

Recall that $d(X, f)$ denotes the number of fixed points of

Lemma 18. Let $H_{1}, H_{2}$ be two finite abelian groups and $f_{i} \in$ $\operatorname{End}\left(H_{i}\right)$. Suppose that $\varphi \in \operatorname{Hom}\left(H_{1}, H_{2}\right)$ is a surjective morphism of dynamical systems. Let $F_{i}=\left\{t \in H_{i}: f_{i}(t)=t\right\}$. Then $\left(H_{1}, f_{1}\right)$ is a fixed point system if and only if the following two conditions are satisfied:

(1) $\left(H_{2}, f_{2}\right)$ is a fixed point system;

(2) $d\left(H_{1}, f_{1}\right)=d\left(\operatorname{ker} \varphi, f_{1}\right) d\left(H_{2}, f_{2}\right)$.

Proof. We write $F_{2}=\left\{y_{i}: 1 \leq i \leq\left|F_{2}\right|\right\}$ and $\varphi\left(x_{i}\right)=y_{i}$. Since $\varphi$ maps cycle vertices into cycle vertices, so the cycle vertices of $\left(H_{1}, f_{1}\right)$ are contained in $\bigcup_{i=1}^{\left|F_{2}\right|}\left(x_{i}+\operatorname{ker} \varphi, f_{1}\right)$, and $d\left(H_{1}, f_{1}\right)=\sum_{i=1}^{\left|F_{2}\right|} d\left(x_{i}+\operatorname{ker} \varphi, f_{1}\right)$. Thus, $\left(H_{1}, f_{1}\right)$ is a fixed point system if and only if $\left(x_{i}+\operatorname{ker} \varphi, f_{1}\right)$ is a fixed point system for any $i=1, \ldots,\left|F_{2}\right|$. By Lemma $17,\left(x_{i}+\operatorname{ker} \varphi, f_{1}\right)$ is a fixed point system if and only if $d\left(x_{i}+\operatorname{ker} \varphi, f_{1}\right)=d\left(\operatorname{ker} \varphi, f_{1}\right)$ and $\left(\operatorname{ker} \varphi, f_{1}\right)$ is a fixed point system. This finishes the proof.

By Theorem 10 we only need to deal with the case that $H$ is a $p$-group. Let $H=\mathbb{Z}_{p^{a_{1}}} \oplus \cdots \oplus \mathbb{Z}_{p^{a_{n}}}$ such that $a_{1} \leq a_{2} \leq$ $\cdots \leq a_{n}$. Recall that any endomorphism of $f$ of $H$ has a matrix representation $A=\left(a_{i j}\right) \in \mathbb{Z}^{n \times n}$, where $p^{a_{i}-a_{j}} \mid a_{i j}$ for $i>j$. If

$$
\left(\begin{array}{c}
u_{1} \\
u_{2} \\
\vdots \\
u_{n}
\end{array}\right)=A\left(\begin{array}{c}
v_{1} \\
v_{2} \\
\vdots \\
v_{n}
\end{array}\right)
$$

then $u_{i} \equiv \sum_{k=1}^{n} a_{i k} v_{i}\left(\bmod p^{a_{i}}\right)$. We also write

$$
\left(\begin{array}{c}
u_{1} \\
u_{2} \\
\vdots \\
u_{n}
\end{array}\right)=A\left(\begin{array}{c}
v_{1} \\
v_{2} \\
\vdots \\
v_{n}
\end{array}\right) \quad\left(\bmod \left(\begin{array}{c}
p^{a_{1}} \\
p^{a_{2}} \\
\vdots \\
p^{a_{n}}
\end{array}\right)\right) \text {. }
$$

We recall the Smith normal form and invariant factor of matrices over a principal ideal ring.
Definition 19. Let $R$ be a principal ideal ring and $A \in R^{n \times n}$. There exist invertible matrices $U, V \in R^{n \times n}$ such that

$$
S=U A V=\left(\begin{array}{ccccc}
s_{1} & & & & \\
& \ddots & & & \\
& & s_{m} & & \\
& & 0 & & \\
& & & \ddots & \\
& & & & 0
\end{array}\right) \text {, }
$$

where $s_{i} \mid s_{i+1}$ for $1 \leq i \leq m-1 . S$ is called the Smith normal form of $A$. The diagonal entries $s_{i}$ are called the invariant factors of $A$ and are unique up to units.

Theorem 20 ([8], Theorem 5.2). Let $p$ be a prime and $H=$ $\mathbb{Z}_{p^{a}}^{n}$ and $A \in \mathbb{Z}^{n \times n}$. Then $(H, A)$ is a fixed point system if and only if the following conditions are satisfied.

(1) $A^{n}(A-I) \equiv 0(\bmod p)$;

(2) Each invariant factors of $A-I$ over $\mathbb{Z}_{p^{a}}$ is a unit or 0.

Corollary 21. Let $H=\mathbb{Z}_{p^{a_{1}}} \oplus \cdots \oplus \mathbb{Z}_{p^{a_{n}}}$ and $A=\left(a_{i j}\right) \in \mathbb{Z}^{n \times n}$, where $a_{1} \leq a_{2} \leq \cdots \leq a_{n}$ and $p^{a_{i}-a_{j}} \mid a_{i j}$ for $i \geq j$. Suppose that $A^{n}(A-I) \equiv 0(\bmod p)$ and each invariant factor of $A-I$ over $\mathbb{Z}_{p^{a_{n}}}$ is a unit or 0 . Then $(H, A)$ is a fixed point system.

Proof. Since the canonical mapping $\varphi:\left(\mathbb{Z}_{p^{a_{n}}}^{n}, A\right) \rightarrow(H, A)$ is a surjective morphism of dynamical systems, it follows from Lemma 18 and Theorem 20 that $(H, A)$ is a fixed point system.

However, the converse of Corollary 21 is not true in general. Consider the following example.

Example 22. Let $A=\left(\begin{array}{ll}1 & 1 \\ 3 & 0\end{array}\right)$ and $H_{1}=\mathbb{Z}_{3} \oplus \mathbb{Z}_{3}, H_{2}=\mathbb{Z}_{9} \oplus \mathbb{Z}_{9}$, and $H=\mathbb{Z}_{3} \oplus \mathbb{Z}_{9}$. By a direct computation, $\left(H_{1}, A\right)$ is a fixed point system with three fixed points $(0,0),(1,0)$, and $(2,0)$, and $\left(H_{2}, A\right)$ has three fixed points $(0,0),(3,0)$, and $(6,0)$, and two 3 -cycle $\{(1,3),(4,3),(7,3)\}$ and $\{(2,6),(8,6),(5,6)\}$, and $(H, A)$ is a fixed point system with 3 fixed points $(0,0),(1,3)$, and $(2,6)$.

Let $A=\left(a_{i j}\right)$ be an integer matrix and $n \in \mathbb{Z}$. We write $n \mid A$ if $n \mid a_{i j}$ for any $i, j$.

Theorem 23. Let $H=\mathbb{Z}_{p^{a_{1}}}^{n_{1}} \oplus \mathbb{Z}_{p^{a_{2}}}^{n_{2}} \oplus \cdots \oplus \mathbb{Z}_{p^{a_{r}}}^{n_{a_{1}}}$ with $a_{1}<$ $a_{2}<\cdots<a_{r}$. Let $T=\left(A_{i j}\right)_{1 \leq i, j \leq r}$ be a matrix with partition $A_{i j} \in \mathbb{Z}^{n_{j} \times n_{i}}$ such that $p^{a_{i}-a_{j}} \mid A_{i j}$ for $i>j$. Let $B_{i j}=p^{a_{j}-a_{i}} A_{i j}$ and

$$
T_{j}=\left(\begin{array}{cccc}
B_{j j} & B_{j j+1} & \cdots & B_{j r} \\
B_{j+1 j} & B_{j+1 j+1} & \cdots & B_{j+1 r} \\
\vdots & \vdots & \vdots & \vdots \\
B_{r j} & B_{r j+1} & \cdots & B_{r r}
\end{array}\right)
$$


for $j=1, \ldots, r$. Let $a_{0}=0$ and $H_{j}=\mathbb{Z}_{p^{a_{j}-a_{j-1}}}^{\sum_{i=1}^{r} n_{i}}$. Then $(H, T)$ is a fixed point system if and only if

(1) $\left(H_{j}, T_{j}\right)$ is a fixed point system for each $j$;

(2) $d(H, T)=\prod_{j=1}^{r} d\left(H_{j}, T_{j}\right)$.

Proof. We induct on $r$. The case $r=1$ is trivial. Let $F_{1}=$ $\mathbb{Z}_{p^{a_{2}-a_{1}}}^{n_{2}} \oplus \mathbb{Z}_{p^{a_{3}-a_{1}}}^{n_{3}} \oplus \cdots \oplus \mathbb{Z}_{p^{a_{r}-a_{1}}}^{n_{r}}$ and

$$
C_{1}=\left(\begin{array}{cccc}
A_{22} & A_{23} & \cdots & A_{2 r} \\
A_{32} & A_{33} & \cdots & A_{3 r} \\
\vdots & \vdots & \vdots & \vdots \\
A_{r 2} & A_{r 3} & \cdots & A_{r r}
\end{array}\right)
$$

Define

$$
\begin{gathered}
\varphi: H \longrightarrow F_{1}, \\
\varphi\left(\begin{array}{c}
X_{1} \\
X_{2} \\
\vdots \\
X_{r}
\end{array}\right) \equiv\left(\begin{array}{c}
X_{2} \\
X_{3} \\
\vdots \\
X_{r}
\end{array}\right)
\end{gathered}
$$

$$
\left(\bmod \left(\begin{array}{c}
p^{a_{2}-a_{1}} \\
p^{a_{3}-a_{1}} \\
\vdots \\
p^{a_{r}-a_{1}}
\end{array}\right)\right)
$$

where $X_{i} \in \mathbb{Z}_{p^{a_{i}}}^{n_{i}}$. Since $p^{a_{i}-a_{1}} \mid A_{i 1}$, one has

$$
\begin{gathered}
\varphi A\left(\begin{array}{c}
X_{1} \\
X_{2} \\
\vdots \\
X_{r}
\end{array}\right) \\
=\left(\begin{array}{c}
A_{21} X_{1}+A_{22} X_{2}+\cdots+A_{2 r} X_{r} \\
A_{31} X_{1}+A_{32} X_{2}+\cdots+A_{3 r} X_{r} \\
\vdots \\
A_{r 1} X_{1}+A_{r 2} X_{2}+\cdots+A_{r r} X_{r}
\end{array}\right) \\
\equiv\left(\begin{array}{c}
A_{22} X_{2}+\cdots+A_{2 r} X_{r} \\
A_{32} X_{2}+\cdots+A_{3 r} X_{r} \\
\vdots \\
A_{r 2} X_{2}+\cdots+A_{r r} X_{r}
\end{array}\right) \equiv C_{1} \varphi\left(\begin{array}{c}
X_{1} \\
X_{2} \\
\vdots \\
X_{r}
\end{array}\right)
\end{gathered}
$$

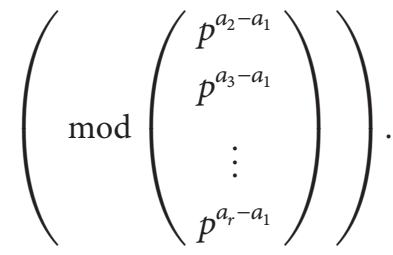

It follows that $\varphi:(H, A) \rightarrow\left(F_{1}, C_{1}\right)$ is a surjective morphism of dynamical systems. By Lemma $18,(H, A)$ is a fixed point system if and only if $\left(F_{1}, C_{1}\right)$ is a fixed point system and $d(H, A)=d(\operatorname{ker} \varphi, A) d\left(F_{1}, C_{1}\right)$. By induction, $\left(F_{1}, C_{1}\right)$ is a fixed point system if and only if $\left(H_{j}, T_{j}\right)$ is a fixed point system for each $j \geq 2$ and $d\left(F_{1}, C_{1}\right)=\prod_{j=2}^{r} d\left(H_{j}, T_{j}\right)$. It remains to show that $(\operatorname{ker} \varphi, A) \simeq\left(H_{1}, T_{1}\right)$, where $T_{1}=$ $\left(p^{a_{j}-a_{i}} A_{i j}\right)_{1 \leq i, j \leq r}$.

We set $\psi: H_{1}=\mathbb{Z}_{p^{a_{1}}}^{\sum_{r}^{r} n_{r}} \rightarrow H$.

$\psi\left(\begin{array}{c}Y_{1} \\ Y_{2} \\ \vdots \\ Y_{r}\end{array}\right) \equiv\left(\begin{array}{c}Y_{1} \\ p^{a_{2}-a_{1}} Y_{2} \\ \vdots \\ p^{a_{r}-a_{1}} Y_{r}\end{array}\right)$

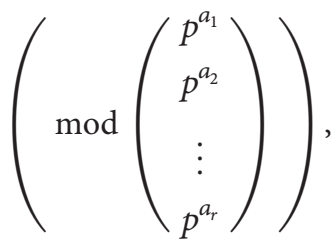

where $Y_{i} \in \mathbb{Z}_{p^{a_{1}}}^{n_{n_{1}}}$. It is clear that $\psi$ is injective and $\operatorname{Im} \psi=\operatorname{ker} \varphi$. We have

$$
\begin{aligned}
& \psi T_{1}\left(\begin{array}{c}
Y_{1} \\
Y_{2} \\
\vdots \\
Y_{r}
\end{array}\right) \\
& =\left(\begin{array}{c}
A_{11} Y_{1}+p^{a_{2}-a_{1}} A_{12} Y_{2}+\cdots+p^{a_{r}-a_{1}} A_{1 r} Y_{r} \\
A_{21} Y_{1}+p^{a_{2}-a_{1}} A_{22} Y_{2}+\cdots+p^{a_{r}-a_{1}} A_{2 r} Y_{r} \\
\vdots \\
A_{r 1} Y_{1}+p^{a_{2}-a_{1}} A_{r 2} Y_{2}+\cdots+p^{a_{r}-a_{1}} A_{r r} Y_{r}
\end{array}\right) \\
& \equiv A \psi\left(\begin{array}{c}
Y_{1} \\
Y_{2} \\
\vdots \\
Y_{r}
\end{array}\right) \quad\left(\bmod \left(\begin{array}{c}
p^{a_{1}} \\
p^{a_{2}} \\
\vdots \\
p^{a_{r}}
\end{array}\right)\right) \text {. }
\end{aligned}
$$

Thus, $\left(H_{1}, \psi\right) \simeq(\operatorname{Im} \psi, A)=(\operatorname{ker} \varphi, A)$. The proof is finished by induction.

Remark 24. By Theorem 23, we can determine whether $\left(H_{j}, T_{j}\right)$ is a fixed point system and also can compute 
$d\left(H_{j}, T_{j}\right)$ be using the Smith normal form of $T-I$. So the problem for determining whether $(H, T)$ is a fixed point system is now reduced to solve the congruence equation

$$
(T-I) X \equiv 0 \quad\left(\bmod \left(\begin{array}{c}
p^{a_{1}} \\
p^{a_{2}} \\
\vdots \\
p^{a_{r}}
\end{array}\right)\right)
$$

Example 25. Let $H=\mathbb{Z}_{2} \times \mathbb{Z}_{4} \times \mathbb{Z}_{16}$ and $T=\left(\begin{array}{lll}1 & 1 & 0 \\ 2 & 2 & 3 \\ 0 & 4 & 2\end{array}\right)$. By Theorem 23, let $T^{\prime}=\left(\begin{array}{ccc}1 & 2 & 0 \\ 1 & 2 & 12 \\ 0 & 1 & 2\end{array}\right)$, and let

$$
\begin{aligned}
& H_{1}=\mathbb{Z}_{2}^{3}, \\
& T_{1}=\left(\begin{array}{ccc}
1 & 2 & 0 \\
1 & 2 & 12 \\
0 & 1 & 2
\end{array}\right)=\left(\begin{array}{lll}
1 & 0 & 0 \\
1 & 0 & 0 \\
0 & 1 & 0
\end{array}\right)(\bmod 2), \\
& H_{2}=\mathbb{Z}_{2}^{2} \text {, } \\
& T_{2}=\left(\begin{array}{ll}
2 & 12 \\
1 & 2
\end{array}\right)=\left(\begin{array}{ll}
0 & 0 \\
1 & 0
\end{array}\right)(\bmod 2), \\
& H_{3}=\mathbb{Z}_{4} \text {, } \\
& T_{3}=(2) \quad(\bmod 4) \text {. }
\end{aligned}
$$

It is straightforward to check that $\left(H_{1}, T_{1}\right)$ is a fixed point system with two fixed points $(0,0,0)^{T},(1,1,1)^{T},\left(H_{2}, T_{2}\right)$ is a fixed point system with one fixed point $(0,0)^{T}$, and $\left(H_{3}, T_{3}\right)$ is a fixed point system with two fixed points 0 . Now we compute the number of fixed points of $(H, T)$. Let $X=(x, y, z)^{T}$ be a fixed point in $(H, T)$. Then $T X=X$ and

$$
\begin{aligned}
y=0 & (\bmod 2), \\
2 x+y+3 z=0 & (\bmod 4), \\
4 y+z=0 & (\bmod 8) .
\end{aligned}
$$

We derive that $y=0,2(\bmod 4)$ and $z=0(\bmod 8)$ by the first and third equation. Hence, the second equation is equivalent to $2 x+y=0(\bmod 4)$. It follows that $x=$ $0(\bmod 2)$ if $y=0(\bmod 4)$, and $x=1(\bmod 2)$ if $y=$ $2(\bmod 4)$. Thus, $(H, T)$ has two fixed points $(0,0,0)^{T}$ and $(1,2,0)^{T}$. By Theorem 23 again, $(H, T)$ is a fixed point system.

\section{Conflicts of Interest}

The authors declare that there are no conflicts of interest regarding the publication of this paper.

\section{Acknowledgments}

This research was supported by the National Natural Science Foundation of China (Grant no. 11371343).

\section{References}

[1] D. Bollman, O. Colón-Reyes, and E. Orozco, "Fixed points in discrete models for regulatory genetic networks," Eurasip Journal on Bioinformatics and Systems Biology, vol. 2007, Article ID 97356, 8 pages, 2007.

[2] J. R. Brown, "Inverse limits, entropy and weak isomorphism for discrete dynamical systems," Transactions of the American Mathematical Society, vol. 164, pp. 55-66, 1972.

[3] B. Elspas, "The theory of autonomous linear sequential networks," in Linear Sequential Switching Circuits, pp. 21-61, Holden-Day, San Francisco, Calif, USA, 1965.

[4] R. A. Hernández Toledo, "Linear finite dynamical systems," Communications in Algebra, vol. 33, no. 9, pp. 2977-2989, 2005.

[5] E. Brown and T. P. Vaughan, "Cycles of directed graphs defined by matrix multiplication $(\bmod n)$, , Discrete Mathematics, vol. 239, no. 1-3, pp. 109-120, 2001.

[6] E. Bach and A. Bridy, "On the number of distinct functional graphs of affine-linear transformations over finite fields," Linear Algebra and its Applications, vol. 439, no. 5, pp. 1312-1320, 2013.

[7] G. Xu and Y. M. Zou, "Linear dynamical systems over finite rings," Journal of Algebra, vol. 321, no. 8, pp. 2149-2155, 2009.

[8] G. Deng, "Cycles of linear dynamical systems over finite local rings," Journal of Algebra, vol. 433, pp. 243-261, 2015.

[9] M. Sha, "Digraph from endomorphism of finite cyclic groups," Journal of Combinatorial Mathematics and Combinatorial Computing, vol. 83, no. 1, pp. 41-58, 2010.

[10] G. Deng and P. Yuan, "On the structure of $G(H, k)$," Algebra Colloquium, vol. 21, no. 2, pp. 317-330, 2014.

[11] C. Lucheta, E. Miller, and C. Reiter, "Digraphs from powers modulo p," The Fibonacci Quarterly, vol. 34, no. 3, pp. 226-239, 1996.

[12] T. D. Rogers, "The graph of the square mapping on the prime fields," Discrete Mathematics, vol. 148, no. 1-3, pp. 317-324, 1996.

[13] M. Sha and S. Hu, "Monomial dynamical systems of dimension one over finite fields," Acta Arithmetica, vol. 148, no. 4, pp. 309331, 2011.

[14] B. Wilson, "Power digraphs modulo n," The Fibonacci Quarterly, vol. 36, no. 3, pp. 229-239, 1998.

[15] J. J. Rotman, An Introduction to the Theory of Groups, vol. 148 of Graduate Texts in Mathematics, Springer, New York, NY, USA, 4th edition, 1995.

[16] T. A. Agafonova and V. F. Gor'kovoi, "Automorphisms of graphs," Journal of Mathematical Sciences, vol. 72, no. 5, pp. $3341-3343,1994$. 


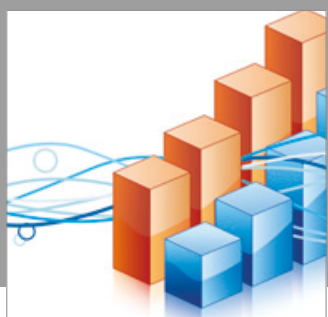

Advances in

Operations Research

vatersals

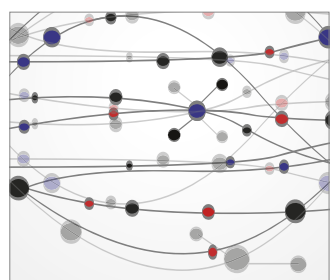

\section{The Scientific} World Journal
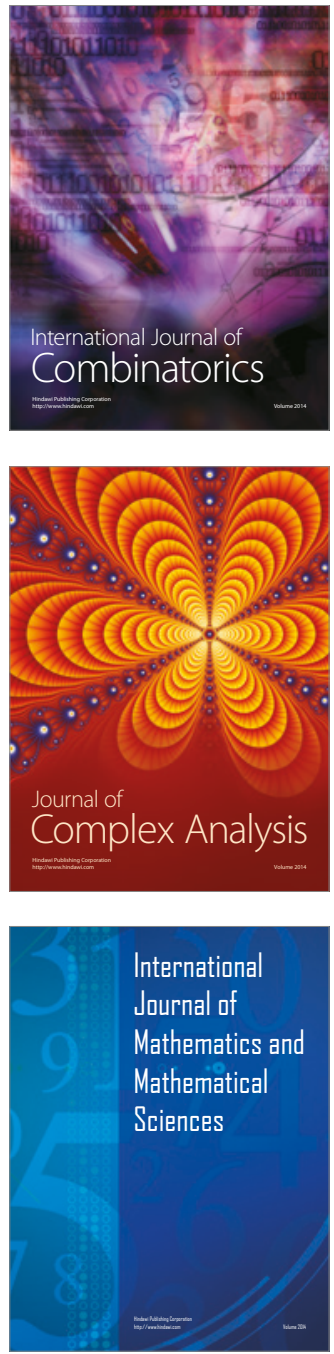
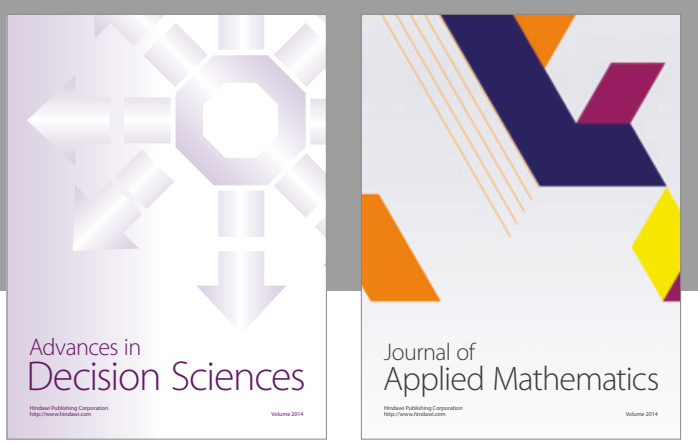

Algebra

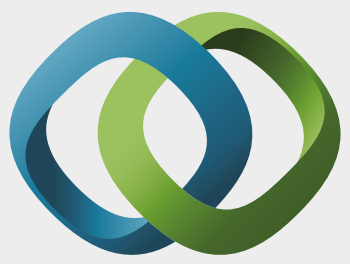

\section{Hindawi}

Submit your manuscripts at

https://www.hindawi.com
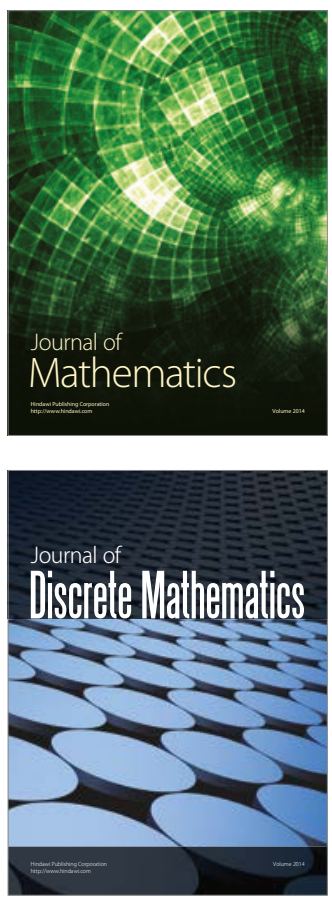

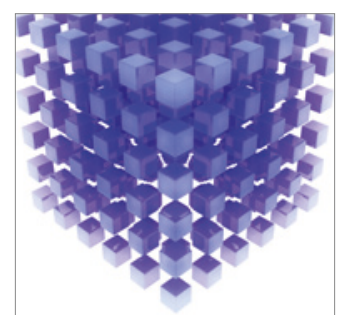

Mathematical Problems in Engineering
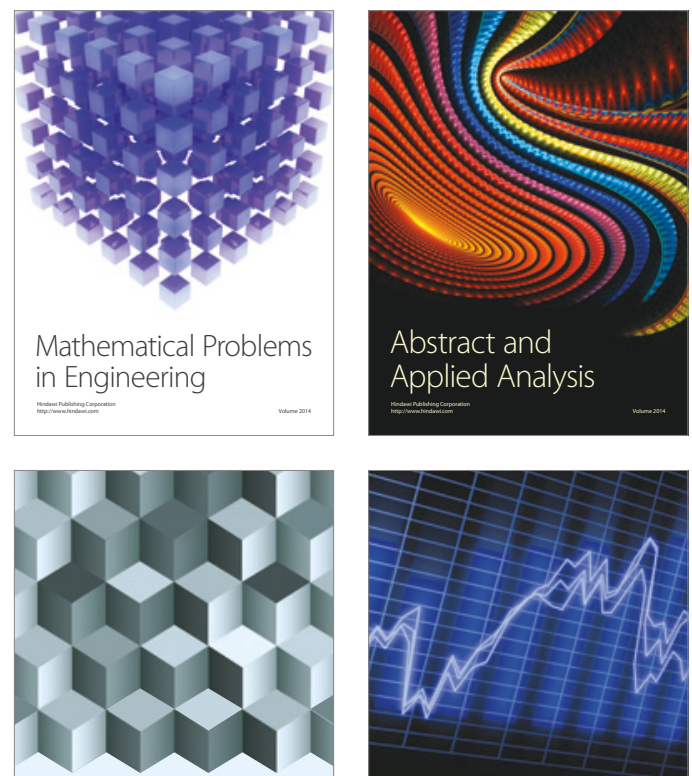

Journal of

Function Spaces

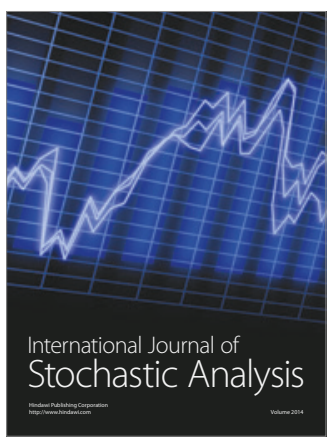

Probability and Statistics
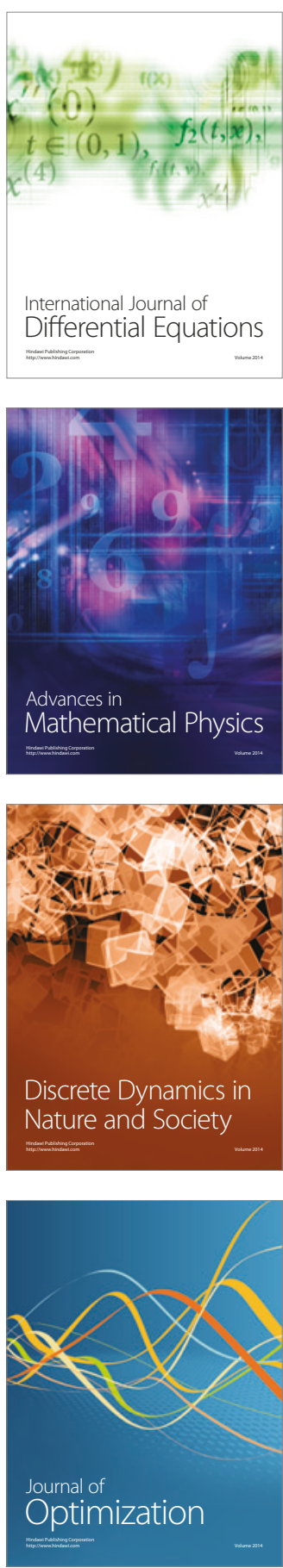